$a_{112}=a_{121}=a_{212}=1$. The trilinear form associated with this matrix is $x_{1} y_{1} z_{2}+x_{1} y_{2} z_{1}+x_{2} y_{1} z_{1}$, which is equivalent to $L$ under the transformations $x_{1}=x_{2}^{\prime}, x_{2}=x_{1}^{\prime}$. Since the matrices of $S$ and $K$ can be taken to be the same, the factorization rank of $S$ is 1 . We have proved the following result.

THEOREM. The factorization ranks of the forms in the sets $(K, S),(R, P, H)$, and $(L, Q)$ are 1,2 , and 3 , respectively.

The equivalence of cubics to $P, Q, S$ can be recognized very simply without the use of factorization rank from the theory of my previous Bulletin paper.

Armour Institute of Technology

\title{
A NOTE ON THE DEGREE OF POLYNOMIAL APPROXIMATION*
}

\section{BY J. H. CURTISS}

Let $C$ be a rectifiable Jordan curve of the finite $z$ plane. We shall say that a function $f(z)$ belongs to the class $\operatorname{Lip}(C, j, \alpha)$ if $f(z)$ is regular in the limited region bounded by $C$ (which we shall call the interior of $C$ ), if $f(z)$ is continuous in the corresponding closed region, and if the $j$ th derivative of $f(z)$ is also continuous in this closed region and satisfies a Lipschitz condition with exponent $\alpha$ on $C$ :

$$
\left|f^{(j)}\left(z_{1}\right)-f^{(j)}\left(z_{2}\right)\right| \leqq M\left|z_{1}-z_{2}\right|^{\alpha}
$$

$z_{1}, z_{2}$ on $C$. The number $\alpha$ will be positive and not greater than unity. The number $j$ will be a positive integer or zero; we define $f^{(0)}(z)$ to be identically $f(z)$. The object of this note is to establish the following existence theorem.

THEOREM. Let the point set $S$ consist of a finite number of closed limited Jordan regions of the z plane bounded by the mutually exterior analytic curves $C_{1}, C_{2}, \cdots, C_{\lambda}$. Let the functions $f_{1}(z), f_{2}(z), \cdots, f_{\lambda}(z)$ belong respectively to the classes

$$
\operatorname{Lip}\left(C_{1}, j, \alpha\right), \quad \operatorname{Lip}\left(C_{2}, j, \alpha\right), \quad \cdots, \quad \operatorname{Lip}\left(C_{\lambda}, j, \alpha\right),
$$

\footnotetext{
* Presented to the Society, December 31, 1935.
} 
and let $f(z) \equiv f_{\nu}(z)$, where $z$ is on and interior to $C_{\nu}$, for $\nu=1,2$, ..., $\lambda$. Then there exist polynomials $p_{n}(z)$ of respective degrees $n{ }^{*}(n=1,2, \cdots)$, such that $f(z)-p_{n}(z)=O\left(n^{-j-\alpha}\right)$ uniformly for $z$ on $S$.

The theorem for the case in which $S$ is the closed interior of the circle $\gamma:|z|=1$ is an immediate consequence of certain results of Bernstein and Jackson on approximation by trigonometric sums. For let the function $f(z)$ belong to the class $\operatorname{Lip}(\gamma, 0, \alpha)$. If we write $z=r e^{i \theta}$ and $f\left(e^{i \theta}\right) \equiv u(\theta)+i v(\theta)$, it is easily verified that both $u(\theta)$ and $v(\theta)$ satisfy Lipschitz conditions with exponent $\alpha$. The work of Bernstein and Jackson $\dagger$ proves that there exist two trigonometric sums of the $n$th order,

$$
\begin{aligned}
& U_{n}(\theta)=\frac{1}{2} d_{n, 0} a_{0}+\sum_{k=1}^{n} d_{n, k}\left(a_{k} \cos k \theta+b_{k} \sin k \theta\right), \\
& V_{n}(\theta)=\frac{1}{2} d_{n, 0} g_{0}+\sum_{k=1}^{n} d_{n, k}\left(g_{k} \cos k \theta+h_{k} \sin k \theta\right),
\end{aligned}
$$

such that uniformly for all values of $\theta, u(\theta)-U_{n}(\theta)=O\left(n^{-\alpha}\right)$ and $v(\theta)-V_{n}(\theta)=O\left(n^{-\alpha}\right)$. The numbers $a_{k}$ and $b_{k}$ are the Fourier coefficients of the function $u(\theta)$ and the numbers $g_{k}$ and $h_{k}$ are the Fourier coefficients of the function $v(\theta)$. Let $f(z)$ $=\sum_{k=0}^{\infty} A_{k} z^{k},|z| \leqq 1$. Then

$$
U_{n}(\theta)+i V_{n}(\theta)=\sum_{k=0}^{n} d_{n, k} A_{k} e^{i k \theta}
$$

and it follows that

$$
f(z)-\sum_{k=0}^{n} d_{n, k} A_{k} z^{k}=O\left(n^{-\alpha}\right)
$$

uniformly for $|z| \leqq 1$. The extension to positive values of $j$ is readily effected by methods similar to those employed by Jackson $\ddagger$ in this connection; the details are left to the reader.

* A polynomial in $z$ of degree $n$ is any expression of the form $a_{0}+a_{1} z$ $+a_{2} z^{2}+\cdots+a_{n} z^{n}$; we do not assume $a_{n}$ to be different from zero.

$\dagger$ Jackson, The Theory of Approximation, Colloquium Publications of this Society, vol. 11, 1930, pp. 1-12; in particular, Theorem IV; Bernstein, Mémoires de l'Académie Royale de Belgique, (2), vol. 4 (1912), pp. 88-89.

$\ddagger$ Jackson, op. cit., pp. 9-11. 
We turn to the general case. Let $w=\psi_{\nu}(z)$ denote an analytic function which maps the interior of the curve $C_{\nu}$ conformally onto the interior of the circle $\gamma:|w|=1$, and let $z=\psi_{\nu}^{-1}(w)$ denote the inverse of this function. A number $R>1$ exists such that the analytic curves $C_{\nu}^{\prime}: \psi_{\nu}(z)=R,(\nu=1,2, \cdots, \lambda)$, are mutually exterior and such that the function $\psi_{\nu}(z)$ is regular and univalent in a limited simply connected region containing the curve $C_{\nu}^{\prime},(\nu=1, \cdots, \lambda)$. By using the fact that the function $\psi_{\nu}^{-1}(w)$ is regular for $|w|<R$, we may easily show that each of the functions $f_{v}\left[\psi_{\nu}{ }^{-1}(w)\right]$ belongs to the class $\operatorname{Lip}(\gamma, j, \alpha)$. Hence for each value of $\nu$ there exist functions $F_{\nu, n}(z)$ that are polynomials in $w=\psi_{\nu}(z)$ of respective degrees $n,(n=1,2, \cdots)$, such that

$$
f_{\nu}(z)-F_{\nu, n}(z)=O\left(n^{-j-\alpha}\right)
$$

uniformly for $z$ on and interior to $C_{\nu}$. The functions $F_{\nu, n}(z)$, $(n=1,2, \cdots)$, are regular in a limited simply connected region containing the curve $C_{\nu}^{\prime}$. Moreover, since $F_{\nu, n}(z)=O(1)$ uniformly for $\left|\psi_{\nu}(z)\right|=1$, it follows from a result of Faber* that $F_{v, n}(z)=O\left(R^{n}\right)$ uniformly for $|\psi(z)| \leqq R$. We define functions $F_{n}(z)$ as follows: $F_{n}(z) \equiv F_{\nu, n}(z), z$ on and interior to $C_{\nu}^{\prime}$, $(n=1,2, \cdots ; \nu=1,2, \cdots, \lambda)$. Then

$$
F_{n}(z)=O\left(R^{n}\right)
$$

uniformly for $z$ on and interior to $C_{\nu}^{\prime},(\nu=1, \cdots, \lambda)$.

Now let $\phi=\phi(z)$ denote an analytic function which maps the complement of $S$ conformally (but not necessarily uniformly) onto the region $|w|>1$ so that the point $z=\infty$ corresponds to the point $w=\infty$. We choose a number $\mu>1$ such that the locus $\Gamma:|\phi(z)|=\mu$ consists of $\lambda$ analytic curves lying respectively interior to the curves $C_{\nu}^{\prime}$ but containing the curves $C_{\nu}$ in their respective interiors. $\dagger$ We also select numbers $\mu_{1}, 1<\mu_{1}<\mu$, and $r, \mu_{1} / \mu<r<1$. The $\lambda$ components of the locus $\Gamma_{1}:|\phi(z)|=\mu$, lie respectively interior to those of the locus $\Gamma$, but contain the curves $C_{\nu}$ in their respective interiors.

* Faber, Münchner Berichte, 1922, pp. 157-178. See also Walsh, Interpolation and Approximation by Rational Functions in the Complex Domain, Colloquium Publications of this Society, vol. 20, 1935, pp. 77-78.

$\dagger$ For the proof that such a number $\mu$ exists, see, for instance, Walsh, op. cit., pp. $65-68$, where the locus $|\phi(z)|=K \geqq 1$ is described in some detail. 
It has been established by Szegö* that there exists on the boundary of $S$ a set of points $z_{k}^{(m)},(k=1,2, \cdots, m+1$; $m=0,1,2, \cdots)$, such that

$$
\left|\omega_{m}(z)\right|^{1 / m} \rightarrow \Delta|\phi(z)|
$$

uniformly for $z$ on any closed point set of the complement of $S$, where $\Delta$ denotes the transfinite diameter of $S$, and where $\omega_{m}(z)=\left(z-z_{1}{ }^{(m)}\right)\left(z-z_{2}{ }^{(m)}\right) \cdots\left(z-z_{m+1}^{(m)}\right)$. The polynomial of degree $m$ which coincides with the function $F_{n}(z)$ in the points $z_{k}{ }^{(m)}$, $(k=1,2, \cdots, m+1)$, may be written as follows:

$$
P_{m}(z)=\frac{1}{2 \pi i} \int_{\Gamma} \frac{F_{n}(t)}{t-z}\left(1-\frac{\omega_{m}(z)}{\omega_{m}(t)}\right) d t,
$$

as may be verified directly; and for $z$ on $\Gamma_{1}$ we have

$$
\left|P_{m}(z)-F_{n}(z)\right| \leqq \frac{1}{2 \pi} \int_{\Gamma}\left|\frac{F_{n}(t)}{t-z} \frac{\omega_{m}(z)}{\omega_{m}(t)}\right||d t| .
$$

By (3) we have

$$
\left|\frac{\omega_{m}(z)}{\omega_{m}(t)}\right|<r^{m}, \quad\left(z \text { on } \Gamma_{1}, t \text { on } \Gamma\right),
$$

for all values of $m$ sufficiently large. Let $d$ denote the minimum of $|t-z|$ for $t$ on $\Gamma, z$ on $\Gamma_{1}$, and let $l$ denote the length of $\Gamma$. Let $m=q n$, where $q$ is a positive integer such that $r_{1}=r^{q} R<1$. From (2), (4), and (5) we obtain

$$
\left|P_{q n}(z)-F_{n}(z)\right| \leqq \frac{M_{1} R^{n}}{d} r^{q n} l=M_{2} r_{1}^{n}, \quad\left(z \text { on } \Gamma_{1}\right),
$$

for all values of $n$ sufficiently large, where $M_{1}$ and $M_{2}$ are independent of $n$ and $z$. By the principle of the maximum, (6) holds for $z$ interior to each of the curves which compose $\Gamma_{1}$. When we combine (6) with (1), we have

$$
f(z)-P_{q n}(z)=O\left(n^{-j-\alpha}+r_{1}^{n}\right)=O\left(n^{-j-\alpha}\right)
$$

* Szegö, Mathematische Zeitschrift, vol. 21 (1924), pp. 205-207; see also Walsh and Russell, Transactions of this Society, vol. 36 (1934), pp. 13-28; Walsh, op. cit., pp. 68-75. The result was proved by Fejér (Göttinger Nachrichten, 1918, pp. 319-331) in the case $\lambda=1$, using methods developed by Hilbert (Göttinger Nachrichten, 1897, pp. 63-70). 
uniformly for $z$ on $S$. We now define the polynomials $p_{n}(z)$ referred to in the statement of the theorem by the following relations: $p_{n}(z) \equiv 0,(n=1,2, \cdots, q-1), p_{q n^{\prime}+h}(z) \equiv P_{q n^{\prime}}(z),(h=0$, $\left.1, \cdots, q-1 ; n^{\prime}=1,2, \cdots\right)$. Since $\left.O\left(n^{\prime-j-\alpha}\right)=O\left[\left(q n^{\prime}+h\right)^{-j-\alpha}\right)\right]$, it follows that $f(z)-p_{n}(z)=O\left(n^{-j-\alpha}\right)$ uniformly for $z$ on $S$, and the proof is complete.

If the smoothness of the function $f(z)$ on the boundary of $S$ is described in terms of a modulus of continuity instead of in terms of a Lipschitz condition, the above methods may be used to derive a result analogous to Theorem IV on page 12 of Jackson's The Theory of Approximation. The following special case is of importance.*

THEOREM. If the function $f(z)$ is regular in each of the regions contained in $S$ and is continuous and possesses a continuous jth derivative for $z$ on $S$, then there exist polynomials $p_{n}(z)$ of respective degrees $n,(n=1,2, \cdots)$, such that $f(z)-p_{n}(z)=O\left(n^{-j}\right)$ uniformly for $z$ on $S$.

The theorem may be used to obtain results on the degree of convergence of certain special sequences of functions. For example, let $C$ be an analytic Jordan curve of the finite plane, let $f(z)$ be a function of the class $\operatorname{Lip}(C, j, \alpha)$, and let polynomials $L_{n}(z)$ of respective degrees $n,(n=0,1,2, \cdots)$, be defined by the requirement of coinciding with the function $f(z)$ in the points $z_{k}{ }^{(n)}=\Phi\left(e^{2 \pi i k /(n+1)}\right),(k=1,2, \cdots, n+1)$, where the function $z=\Phi(w)$ maps the unlimited region bounded by $C$ onto the region $|w|>1$ so that the point $w=\infty$ corresponds to the point $z=\infty$. A previous result of the author $\dagger$ expresses the degree of convergence of the sequence $\left\{L_{n}(z)\right\}$ in terms of that of an arbi. trary sequence of polynomials. This result, when combined with the first theorem of the present paper, yields $\ddagger$ the equations

* The theorem for the case $j=0$ is included in a theorem of Walsh; see Walsh, op. cit., p. 47, Theorem 15.

$\dagger$ J. H. Curtiss, Transactions of this Society, vol. 38 (1935), pp. 458-473; p. 467 .

$\mp$ This application of the theorem is analogous to Jackson's application of the results in pp. 1-12 of his book to the determination of the degree of convergence of trigonometric sums interpolating to a real function $F(x)$ in equally spaced points. These sums exhibit the degree of convergence involved in (8) 


$$
f(z)-L_{n}(z)=O\left(n^{-j-\alpha}\right)
$$

uniformly for $z$ on an arbitrary closed point set $T$ interior to $C$ and

$$
f(z)-L_{n}(z)=O\left(n^{-j-\alpha} \log n\right)
$$

uniformly for $z$ on $C$.

We may also obtain the degree of convergence of certain sequences of Riemann sums which tend to the Cauchy integral of the function $f(z)$ over the curve $C$. If we write

$$
\sigma_{n}(z)=\sum_{k=1}^{n+1} \frac{f\left(z_{k}^{(n)}\right)}{z-z_{k}^{(n)}}\left(z_{k+1}^{(n)}-z_{k}^{(n)}\right),
$$

then it is possible to show that*

$$
\sigma_{n}(z)-2 \pi i L_{n}(z)=O\left(\rho^{n}\right)
$$

uniformly for $z$ on $T$, where $\rho<1$. Cc.mparison of (7) with (9) indicates that $\dagger$

$$
\sigma_{n}(z)-\int_{C} \frac{f(t)}{t-z} d t=O\left(n^{-j-\alpha}\right)
$$

uniformly for $z$ on $T$.

The Johns Hopkins University

when the function $F(x)$ has properties corresponding to those of a function of class $\operatorname{Lip}(C, j, \alpha)$.

W. E. Sewell (this Bulletin, vol. 41 (1935), pp. 111-117) has recently shown that the partial sums of the expansion of a function $f(z)$ which is of class Lip $(C, j, \alpha)$ in the Faber polynomials belonging to the region interior to $C$ also exhibit the degree of convergence involved in (8) for $z$ on $C$.

* This equation may be obtained by methods used by Curtiss, loc. cit.; see in particular pp. 460-464 and p. 466.

$\dagger$ For further results on degree of convergence of Riemann sums, see Pólya and Szegö, Aufgaben und Lehrsätze, vol. 1, 1925, pp. 35-37, 194-195; and see Walsh and Sewell, this Bulletin, vol. 42 (1936), p. 489, Abstract 284. 